\title{
Primary colon natural killer (NK)/T-cell lymphoma, nasal type, with perforations: a case report and literature review
}

\author{
Yuankang Xie ${ }^{1 \#}$, Yuwen Liu ${ }^{2 \#}$, Chao Wen ${ }^{3}$, Wenlong $\mathrm{Li}^{4}$, Xiaoliang Lu ${ }^{4}$, Longying Deng ${ }^{5}$ \\ ${ }^{1}$ Department of Hepatobiliary Surgery, The First Affiliated Hospital of Gannan Medical University, Ganzhou, China; ${ }^{2}$ Department of General \\ Surgery, The First Affiliated Hospital of Jiangxi Medical College, Shangrao, China; ${ }^{3}$ School of Nursing, Gannan Medical University, Ganzhou, \\ China; ${ }^{4}$ Department of General Surgery, The People's Hospital of Ningdu County, Ganzhou, China; ${ }^{5}$ Department of Gastrointestinal Surgery, The \\ First Affiliated Hospital of Gannan Medical University, Ganzhou, China \\ \#These authors contributed equally to this work. \\ Correspondence to: Longying Deng. Department of Gastrointestinal Surgery, The First Affiliated Hospital of Gannan Medical University, Ganzhou, \\ China. Email: denglongying235@163.com.
}

\begin{abstract}
Extranodal natural killer (NK)/T-cell lymphoma-nasal type (ENKTL-NT) is a rare, aggressive subtype of non-Hodgkin's lymphoma associated with Epstein-Barr virus (EBV) infection and has a poor prognosis. ENKTL-NT primarily involves the nasal cavity, and the colon as the primary site has rarely been reported. Its lack of a characteristic clinical presentation makes early diagnosis difficult to diagnose early, and misdiagnosis is common without the use of immunohistochemistry of specimens. To further understand this rare solid tumor, we report a case in a 51-year-old male patient admitted to hospital with abdominal pain as the primary symptom. A provisional diagnosis of gastrointestinal perforation was made on the basis of enhanced computed tomography of the abdomen, and emergency surgery was performed. However, 58 days after discharge, he suffered a second colonic perforation, underwent emergency surgery and was diagnosed with primary colonic ENKTL-NT based on the immunohistochemical results of the surgical specimen. He was transferred to the oncology department for chemotherapy after recovery from surgery, with gemcitabine, oxaliplatin, and pegaspargase as the chemotherapy regimen. To date, he has completed 11 courses of chemotherapy, and is now in a significantly improved general condition with no signs of tumor recurrence. We also reviewed and compared the literature related to primary colonic ENKTL-NT.
\end{abstract}

Keywords: Colonic perforation; extranodal NK/T-cell lymphoma nasal type; ulcers; case report

Submitted Sep 16, 2021. Accepted for publication Nov 18, 2021.

doi: $10.21037 / \mathrm{apm}-21-3178$

View this article at: https://dx.doi.org/10.21037/apm-21-3178

\section{Introduction}

Natural killer (NK)/T-cell lymphoma is a subtype of non-Hodgkin's lymphoma and is a rare and aggressive malignancy with a poor clinical prognosis (1). Its pathogenesis is complex, and Epstein-Barr virus (EBV) infection is thought to be the direct driver of its development (2). In the 2016 revised version, the World Health Organization classified NK/T-cell lymphoma as a lymphoproliferative disease, with extranodal NK/ T-cell lymphoma (ENKTL-NT) being the relatively most common form (3). ENKTL-NT begins with abnormal transformation and proliferation of EBV-infected NK cells or T-cells (4). More than 70\% of ENKTL-NT cases occur in the nose and the tumor is confined to the adjacent area, but can occur at other sites (5). In an epidemiological study, it was noted that the distribution of ENKTL-NT subtypes did not correlate with country geography or ethnicity (6).

Primary colonic ENKTL-NT is very rare and difficult to identify in the early stages due to the lack of characteristic clinical symptoms and other evidence. Although histologically ENKTL-NT presents as an ulcerative granuloma, differential diagnosis by endoscopic examination is difficult because of other benign 

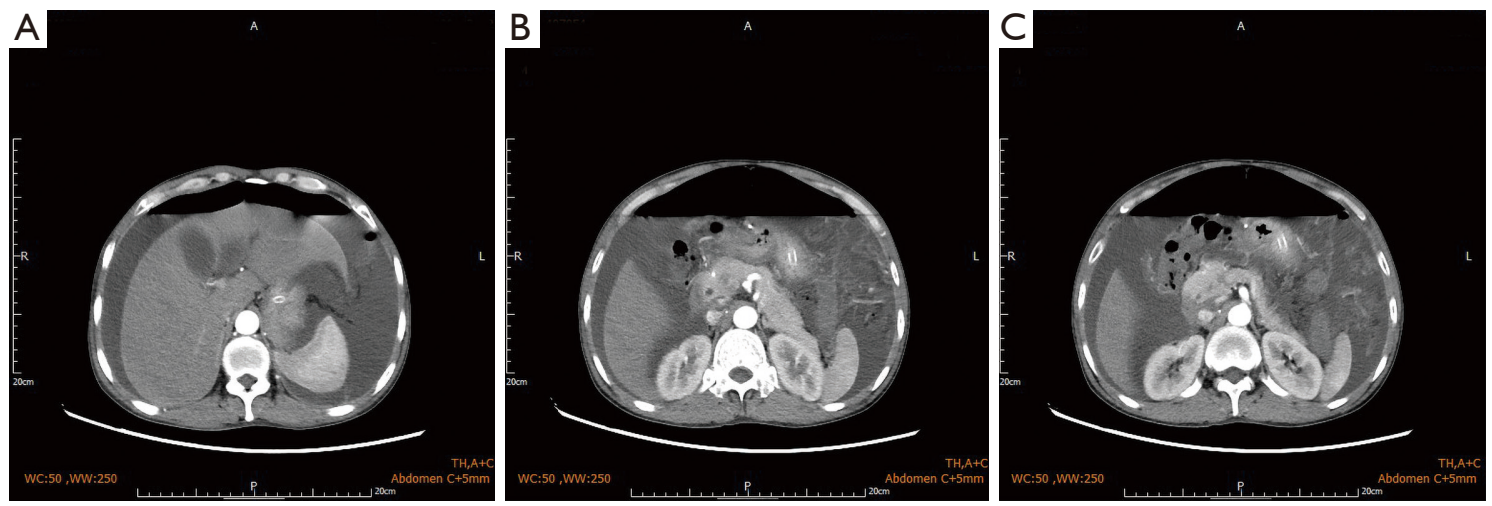

Figure 1 Contrast-enhanced abdominal computed tomography before the first surgery. (A-C) Massive fluid and gas accumulation in the abdominopelvic cavity and retroperitoneum with inflammatory peritoneal changes, suggesting gastrointestinal perforation.

granulomatous proliferative diseases of the colon and malignant neoplasms, which can easily lead to misdiagnosis, and the final diagnosis depends on immunohistochemical analysis of tissue specimens (5). Here, we present a rare case of primary colonic ENKTL-NT, in accordance with the CARE reporting checklist (available at https://dx.doi. org/10.21037/apm-21-3178).

\section{Case presentation}

A 51-year-old male patient presented with the complaint of abdominal pain without obvious cause 1 week previously, located in the mid-upper abdomen and described as persistent vague pain with nausea. He was admitted to the local hospital and treated with antibiotics empirically, but the abdominal pain was not relieved. After 2 days of treatment, the pain gradually increased and involved the whole abdomen, together with constipation and flatulence. He had pain in the lower back, as well as edema in both lower legs without vomiting, fever, or chills. He was referred to us. On admission to the First Affiliated Hospital of Gannan Medical University, physical examination showed pain throughout the abdomen, disappearance of bowel sounds, with positive mobile turbid sounds, and moderate sunken edema in both lower legs. He had a past medical history of gastritis.

Laboratory tests showed an increased leukocyte count and neutrophil ratio, with blood albumin of only $15.9 \mathrm{~g} / \mathrm{L}$. Contrast-enhanced computed tomography (CT) scan of the whole abdomen showed a large amount of fluid and inflammation in the abdominopelvic cavity and retroperitoneum (Figure 1A-1C). The initial diagnosis was a perforated gastrointestinal tract, and emergency surgery was performed. We located a perforation of approximately $1.5 \mathrm{~cm}$ in diameter in the middle section of the transverse colon surrounded by partially ischemic and necrotic omentum, with edema of the intestinal wall around the perforation. There were large amounts of yellow pus partially mixed with feces in the abdominal cavity and white pus covering the surface of the edematous mesentery, small intestine, colon, stomach, liver, and gallbladder. During surgical treatment with a transverse colostomy, the intestinal wall around the perforation and the necrotic omentum were removed for tissue biopsy. Pathology of the specimen showed vasodilatation, inflammatory cell infiltrate, focal point of necrosis, and numerous multinucleated giant cell aggregates. The patient was discharged after symptomatic supportive treatment that included albumin supplementation.

After 58 days, the patient was admitted again to hospital with acute epigastric pain. CT scan of the abdomen showed fluid accumulation in the abdominopelvic cavity and thickening of the intestinal wall (Figure $2 A-2 C$ ). Emergency surgery was again performed, during which we found a large amount of yellow intestinal fluid mixed with food residue filling the abdominal cavity, and a rupture of the intestinal wall in the middle of the transverse colon about $6 \mathrm{~cm}$ from the original fistula. The rupture hole was about one-third of the diameter of the intestinal canal, there was no obvious thickening or ulceration of the canal, and a hard fecal stone was located at its (Figure 3A). Surgical treatment comprised a double lumen fistula at the end of the ileum and a partial resection of the transverse colon, which was successful. Histology of the resected colonic specimen showed focal 

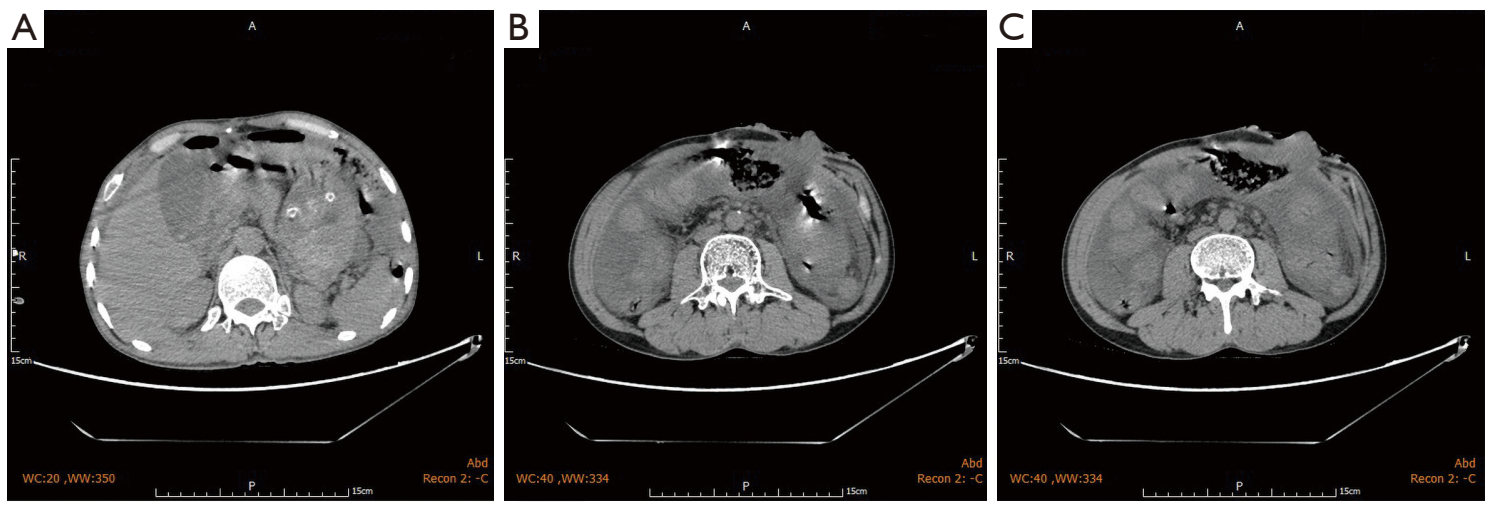

Figure 2 Abdominal computed tomography during second hospitalization. (A-C) computed tomography scan of the abdomen before the second surgery showing peritoneal and pelvic fluid accumulation along with inflammatory peritoneal changes suggesting gastrointestinal perforation.
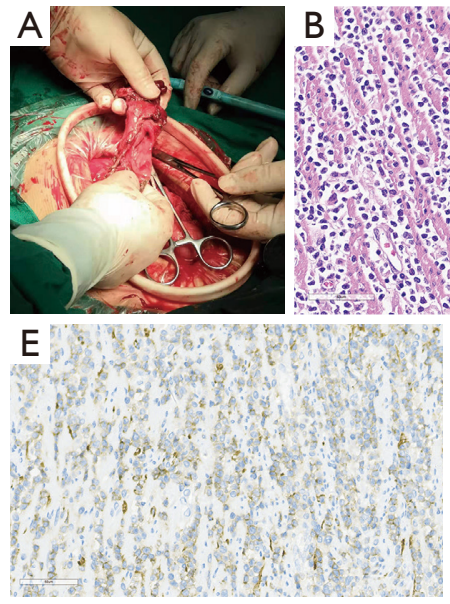
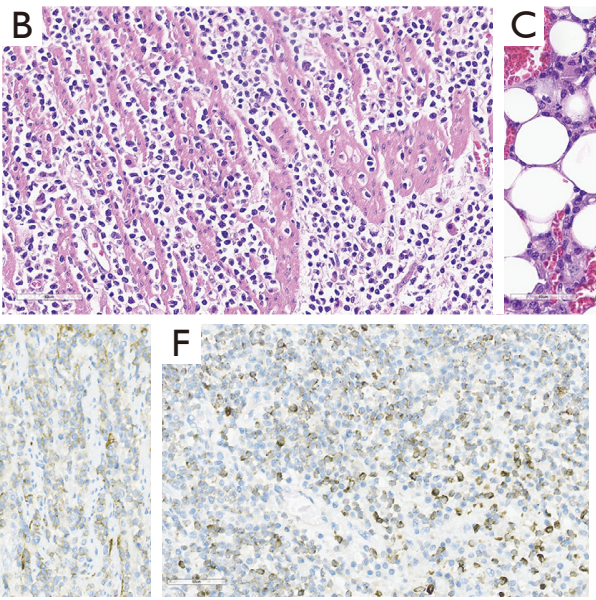
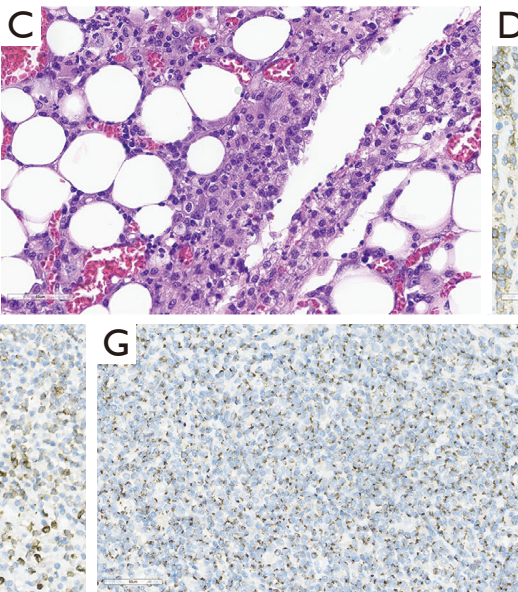
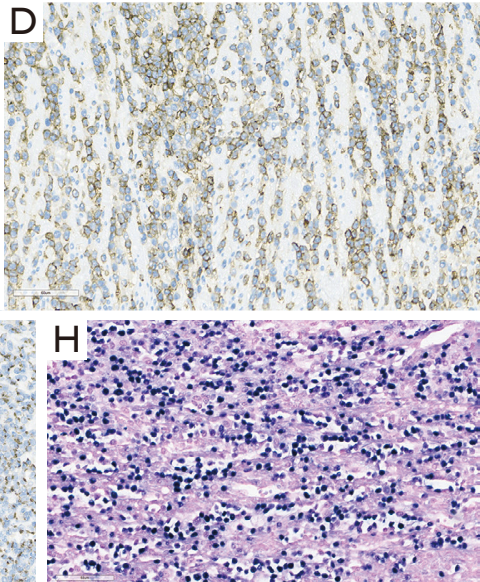

Figure 3 Histological and immunohistochemical findings of the operative biopsy specimens. (A) Perforated colon. (B,C) Tumor tissues showing large number of lymphocytes infiltrating the colonic wall ( $\times 40$, hematoxylin-eosin staining). Immunohistochemical stains showing the tumor cells positive for (D) CD43 (×200), (E) CD56 (×200), (F) GB (×200), (G) TIA-1 (×200) and (H) EBV-encoded small RNA ( 200$)$. EBV, Epstein-Barr virus; GB, granzyme B.

ulcerative granuloma formation in the intestinal wall with massive lymphocytic infiltration of the colonic musculature (Figure $3 B, 3 C$ ). Subsequently, immunohistochemical analysis showed that CD56, CD2, granzyme B (GB), TIA-1, CD43, and CD3 were positive (Figure $3 D-3 H$ ), CD20, $\mathrm{CD} 8$, and $\mathrm{CD} 38$ were partially positive, and $\mathrm{CD} 5, \mathrm{CD} 7$, $\mathrm{CD} 4, \mathrm{CD} 57$, and $\mathrm{CD} 30$ were negative. The $\mathrm{Ki}-67$ positive labeling rate was $\sim 70 \%$. An in situ hybridization (ISH) study revealed positive EBV-encoded RNA (EBER) in tumor cell nuclei. The patient was diagnosed with ENKTL-NT. On the 11th day after the repeat surgery, repeat abdominal CT showed a significant reduction of fluid in the abdomen and pelvis (Figure 4). After a postoperative evaluation, he was transferred to the oncology department for chemotherapy with P-Gmox: gemcitabine, oxaliplatin, and pegaspargase. During chemotherapy patients can experience decreased white blood cell count and neutrophil ratios, and abnormal coagulation, which can induce surgical incision infections. Recombinant human granulocyte growth factor injection was used to promote neutrophil production, and cefotaxime injection was used for antibacterial therapy. On discharge from the hospital, the patient's general condition improved significantly, and the CT scanning showed no signs of tumor recurrence and resolution of abdominal inflammation 

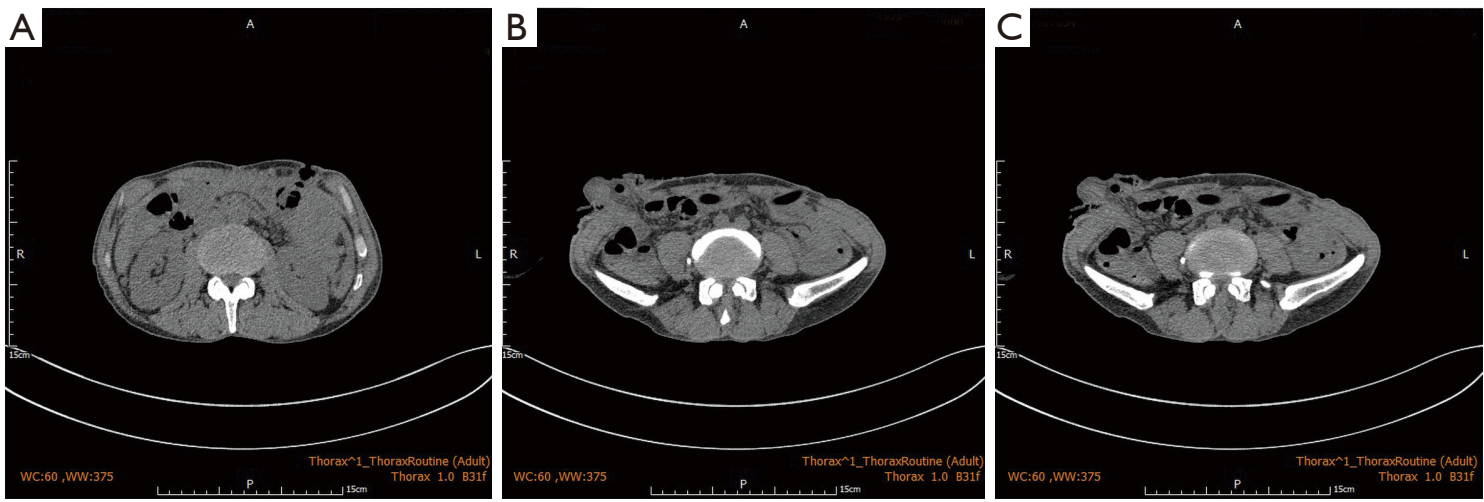

Figure 4 Abdominal computed tomography after the second surgery showing a largely absorbed fluid in the abdomen and pelvis.
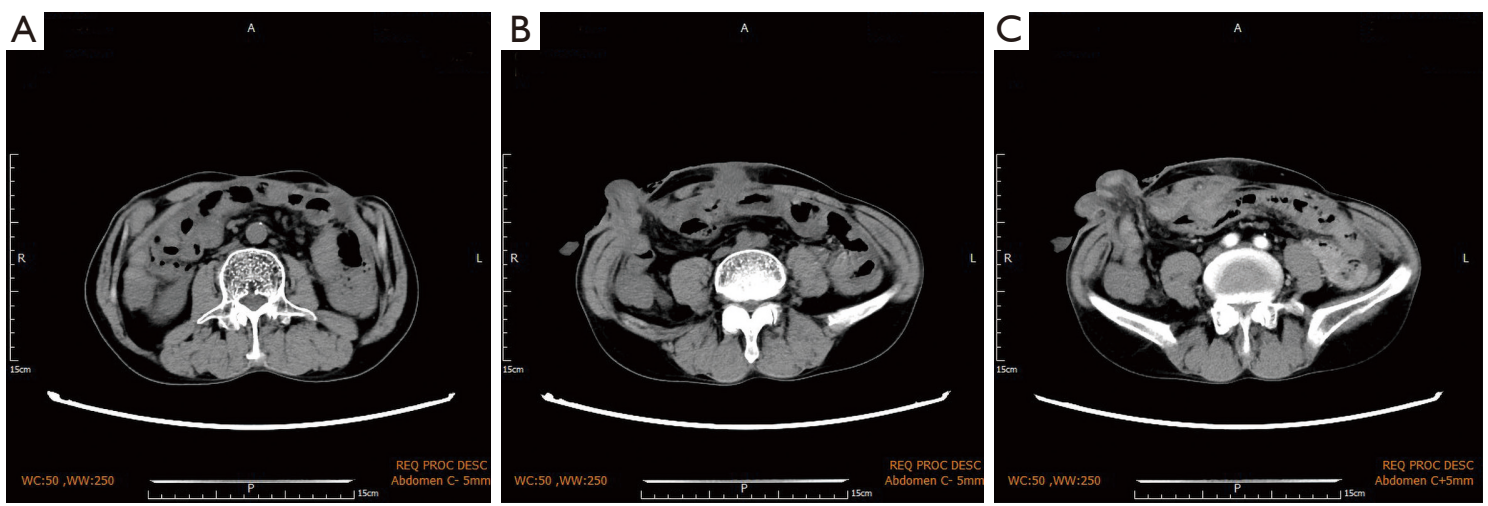

Figure 5 Abdominal computed tomography showing post-operative changes in the abdomen after chemotherapy without any signs of tumor recurrence.

(Figure 5A-5C). To date, he has completed a total of 11 courses of chemotherapy and is still being followed up.

The study was approved by the Ethics Committee of the First Affiliated Hospital of Gannan Medical University (No. LLSC-2021111201), and all procedures involving human participants followed the Declaration of Helsinki (as revised 2013). Written informed consent was obtained from the patient for publication of this case report and accompanying images. A copy of the written consent is available for review by the editorial office of this journal.

\section{Discussion}

ENKTL-NT is an extremely rare form of malignant lymphocytoma. Its prevalence varies widely by geographic region, being relatively more common in Asia and Latin America, and men in their 40s and 50s being more likely to be affected $(2,5,7)$. As a subtype of non-Hodgkin's lymphoma, ENKTL-NT is highly aggressive and has a poor prognosis, with a 5 -year overall survival (OS) rate of $40-50 \%$ (8). The pathogenesis of ENKTL-NT is complex, because EBV infection is closely associated with the development of the tumor. EBV is a ubiquitous human gamma herpesvirus that tends to infect B cells and contribute to their proliferation, and also affects NK cells, T cells, and epithelial cells. EBV DNA persists as a clonally recombinant form without integration in the host genome. Overexpression of the oncogenic protein EBV latent membrane protein-1 (LMP-1) promoting cytokine secretion and thus activating the PI3K/Akt, NF- $\mathrm{KB}$, and JAK/STAT pathways is a possible underlying pathogenic mechanism $(2,9)$. Using high-throughput sequencing technology, deletion of chromosome 6Q leading to mutations in oncogenes such as PRDM1, FOXO3, and PTPRK is also considered to be an important molecular event leading to the occurrence of ENKTL-NT (10). Early studies showed 
mutations in tumour suppressor genes in ENKL, with FAS mutations detected in $50 \%$ of ENKL patients and TP53 mutations in $<50 \%$ (10). ENKTL-NT occurs primarily in the nasal cavity, and colonic primary cases are even rarer. A MEDLINE search revealed 9 unique cases of colonic primary ENKTL-NT-NT (Table 1) (11-19) (including the case reported here). In this case report, although the patient was eventually diagnosed with ENKTL-NT, the presenting symptoms were unusual. We reviewed the existing literature for a more in-depth analysis.

To date, there are no clearly defined clinical manifestations of primary ENKTL-NT in the colon. A study of intestinal primary ENKTL-NT reported by Yang et al. (20) in 2018 showed that compared to ENKTLNT with nasal presentation (age of onset between 40 and 50 years), patients with intestinal primary occurred at a younger age, with a median age of 39 years, nearly $70 \%$ of patients were male, and the majority of cases were located in the colon. The clinical signs included abdominal pain, fever, and intestinal bleeding, with only $23.08 \%$ of patients presenting with diarrhea. Physical examination was almost always non-definitive. Of note, among the 13 cases reported in the study, 6 that occurred in the colon essentially developed intestinal perforation. We suspect the thinness of the colon wall contributes to the development of perforation by proliferating tumor cells invading blood vessels and myocytes, randomly in one or more locations in the affected intestinal wall. It was recently reported that a patient with ENKTL-NT of primary colonic origin developed perforations at multiple sites in the colon during hospitalization (12). In the present case, the patient was initially admitted to the local hospital for abdominal pain only, and fever and diarrhea were excluded. We have no evidence whether the patient had a perforation of the colon before the onset of abdominal pain, but in most cases, the abdominal pain occurred before perforation. Laboratory investigations were not considered to be diagnostic, although lactate dehydrogenase ( $\mathrm{LDH})$ above the normal physiological range was specifically highlighted in a few cases, but the sample size was insufficient for statistical analysis $(15,19,21)$. Similarly, imaging studies were unremarkable, with localized thickening of the intestinal wall insufficient to raise suspicion of ENKTL-NT (12). Endoscopy plays an important role in the identification of intestinal tumors, yet it may be unrewarding in the diagnosis of primary ENKTL-NT of the colon. Several studies have reported that some patients with early colonic primary ENKTL-NT had one or more irregularly sized ulcers in the colon when colonoscopy was performed for abdominal pain and/or intestinal bleeding. Histological examination merely suggests chronic inflammation of the colon and proliferation of lymphocytes, which is nonspecific $(12,15,21)$. In our reported patient, the abdominal pain was sudden and no ulcer was found in whole colon endoscopy, which affected our judgment of his condition. On admission, contrast-enhanced CT of the abdomen showed signs of intestinal perforation and histopathologic examination of specimens from the surgical treatment confirmed severe edema, the lack of an ulcer and suggested inflammation. It is these reasons that lead us to misdiagnose patients. Unfortunately, the patient suffered another gastrointestinal perforation and repeat emergency surgery also revealed no ulceration or thickening of the intestinal wall at the site of the rupture, although a hard fecal mass at the site may have been the "culprit". Our literature search of cases of colonic primary ENKTL-NT complicated by intestinal perforation confirmed the lack of a clarified interpretation of the relationship between perforation site and ulcer. We propose that the full thickness of the colon was invaded by heterogeneous proliferating lymphocytes as well as by the formation of local necrotizing tissue ulcers involving the surrounding intestinal wall tissue. Furthermore, tumor cell invasion of the muscular and submucosal vessels leads to regional ischemic necrosis of the tissue, and inflammatory cell infiltration induces edema that together promotes perforation of the intestinal wall tissue around the ulcer.

Given the absence of typical clinical manifestations, the diagnosis of patients with primary ENKTL-NT of the colon is very difficult, especially in the early stages. Most patients are only diagnosed by pathological findings after surgical resection, but it is already too late. The results of colonoscopy can easily mislead, as primary ENKTL-NT of the colon can easily masquerade as inflammatory bowel disease (IBD), in particular Crohn's disease. The endoscopic findings of single or multiple irregular ulcers combined with histology suggestive of chronic inflammation in the colon make the diagnosis of Crohn's disease appear to be correct. Because the anomalous lymphocytes are located in the submucosal muscular layer of the colon, multiple and deeper tissue biopsies are needed to provide a more accurate diagnosis. In our summary of 9 cases, 5 were misdiagnosed as IBD (Table 1). Clinicians need to be aware that colonic primary ENKTL-NT should be considered when irregular ulcers are found on colonoscopy. Currently, histological examination and immunohistochemistry are the only methods for the diagnosis of ENKTL-NT. Histology 
Table 1 Cases of colonic primary ENKTL-NT-NT in MEDLINE search

\begin{tabular}{|c|c|c|c|c|c|c|c|c|c|c|c|c|c|c|}
\hline Reference & $\begin{array}{l}\text { Sex/age, } \\
\text { years }\end{array}$ & Location & Complication & Clinical manifestation & Misdiagnosis & $\begin{array}{l}\text { Colonic } \\
\text { perforation }\end{array}$ & Morphologic features & Immunophenotype & $\begin{array}{l}\text { TCR gene } \\
\text { rearrangement }\end{array}$ & $\begin{array}{l}\text { ISH of } \\
\text { EBER }\end{array}$ & $\begin{array}{l}\text { Emergency } \\
\text { surgery }\end{array}$ & Treatment & Metastasis & OS (median) \\
\hline $\begin{array}{l}\text { Kim } \\
\text { et al. (11) }\end{array}$ & $M / 38$ & Colon & No & Fever, diarrhea, Gl bleeding & Yes & Yes & $\begin{array}{l}\text { Small and medium sized atypical } \\
\text { lymphocyte infiltration }\end{array}$ & $\begin{array}{l}\text { CD45+, CD45RO+, } \\
\text { CD3+, CD56+ }\end{array}$ & Negative & NM & Yes & CHOP & $\begin{array}{l}\text { Lymph nodes, } \\
\text { liver }\end{array}$ & 3 months \\
\hline $\begin{array}{l}\text { Zheng } \\
\text { et al. (12) }\end{array}$ & $M / 37$ & Colon & Crohn's disease & $\begin{array}{l}\text { Fever, abdominal pain, diarrhea, } \\
\text { Gl bleeding }\end{array}$ & Yes & No & $\begin{array}{l}\text { Small and medium sized atypical } \\
\text { lymphoid cells diffuse proliferation }\end{array}$ & $\mathrm{CD} 56+, \mathrm{CD} 3+, \mathrm{GB}+$ & NM & Positive & No & $\begin{array}{l}\text { 1. Surgery, 2. CHOP, } \\
\text { 3. SMILE }\end{array}$ & Lymph nodes & 2 months \\
\hline $\begin{array}{l}\text { Tseng } \\
\text { et al. (13) }\end{array}$ & $M / 63$ & Colon & Colon adenocarcinoma & Gl bleeding & No & No & Large pleomorphic lymphoid cells & $\begin{array}{l}\mathrm{CD} 3+, \mathrm{CD} 56+, \mathrm{TIA}-1+, \\
\mathrm{CD} 30+\end{array}$ & NM & Positive & No & $\begin{array}{l}\text { 1. Surgery, 2. CHOP, } \\
\text { 3. SMILE }\end{array}$ & $\begin{array}{l}\text { Lymph nodes, } \\
\text { lung }\end{array}$ & 4.5 months \\
\hline $\begin{array}{l}\text { Mahuad } \\
\text { et al. (14) }\end{array}$ & $M / 52$ & Colon & No & Fever & No & No & High-grade lymphocyte infiltration & $\begin{array}{l}\text { CD3+, CD2+, CD56+, } \\
\text { GB+, perforin+, Ki-67: } \\
40 \%\end{array}$ & NM & Positive & No & $\begin{array}{l}\text { 1. Surgery, 2. DA- } \\
\text { EPOCH, 3. SMLLE }\end{array}$ & Lymph nodes & 2 months \\
\hline $\begin{array}{l}\text { Fares } \\
\text { et al. (16) }\end{array}$ & $\mathrm{M} / 43$ & Colon & No & $\begin{array}{l}\text { Fever, abdominal pain, diarrhea, } \\
\text { Gl bleeding, weight loss }\end{array}$ & No & No & NM & $\begin{array}{l}\mathrm{CD} 3+, \mathrm{CD} 4+, \mathrm{CD} 8+, \\
\mathrm{CD} 56+, \mathrm{GB}+\end{array}$ & NM & Positive & No & AspaMetDex & No & 5 months \\
\hline Liet al. (17) & $F / 40$ & Colon & No & Fever, diarrhea & Yes & No & Heterotypic lymphocyte infiltration & $\begin{array}{l}\text { CD3+, TIA-1+, CD56+/-, } \\
\text { Ki-67: } 40 \%\end{array}$ & NM & Positive & No & NM & Lymph nodes & Few months \\
\hline $\begin{array}{l}\text { Duan } \\
\text { et al. (18) }\end{array}$ & $\mathrm{M} / 12$ & Colon & No & Fever, abdominal pain & No & Yes & $\begin{array}{l}\text { Medium-sized atypical lymphoid } \\
\text { cell infiltration }\end{array}$ & $\begin{array}{l}\text { CD3+, CD56+, CD5+, } \\
\text { CD2+, CD8+, CD4+, } \\
\text { CD43+, TIA-1+, GB+, Ki- } \\
67: 60 \%\end{array}$ & Negative & Positive & Yes & NM & No & 1 month \\
\hline $\begin{array}{l}\text { Zhang } \\
\text { et al. (19) }\end{array}$ & $\mathrm{M} / 35$ & Colon & No & Abdominal pain, diarrhea & No & Yes & Small lymphocyte cell infiltration & $\begin{array}{l}\mathrm{CD} 3+, \mathrm{CD} 2+, \mathrm{CD} 43+, \\
\mathrm{CD} 30+, \mathrm{GB}+, \mathrm{TIA}-1+, \mathrm{Ki}- \\
67: 60 \%\end{array}$ & Positive & Positive & Yes & $\begin{array}{l}\text { ED, Gemcitabine, } \\
\text { PD } 1 \text { blockade }\end{array}$ & No & Follow up \\
\hline Our case & $M / 51$ & Colon & No & Abdominal pain & Yes & Yes & Large lymphocytic infiltration & $\begin{array}{l}\text { CD56+, CD2+, GB+, } \\
\text { TIA-1+, CD43+, CD3+, } \\
\text { Ki-67: 70\% }\end{array}$ & NM & Positive & Yes & P-GMOX & No & Follow up \\
\hline
\end{tabular}

(n) 
shows a proliferation of heterogeneous lymphocytes that can accumulate throughout the intestinal wall. In addition, in the myenteric tissue, these heterogeneous lymphocytes infiltrate invasively into the vascular centre to induce massive coagulative necrosis and granulation tissue formation. In general, the tumor cells are derived from NK cells, and immunohistochemistry will be positive for $\mathrm{CD} 56, \mathrm{CD} 2$, and cytoplasmic $\mathrm{CD} 3 \varepsilon$, granzyme B, TIA-1, and perforin. However, in rare cases ENKTLNT can also be of T-cell origin, whereby the tumor cells are negative for CD56 expression, but the T-cell receptor (TCR) gene is rearranged on the T-cell surface. In contrast to other intestinal lymphocytomas, almost all ENKTL-NT cases are associated with EBV infection, with positive ISH for EBV-encoded small RNA (EBER) $(5,10)$. Therefore, it is essential to detect tumor cells expressing CD56 accompanied by positive EBER for the diagnosis of ENKTL-NT plus a positive Ki-67 labeling rate in response to the aggressiveness of the tumor.

In the present case, the diagnosis of primary ENKTLNT in the colon was based on the 3 criteria:

(I) The tumor cells initially appeared in the colon rather than in other anatomical locations;

(II) A history of previous ENKTL-NT-related disease was excluded;

(III) Histological, immunohistochemical, and in situ molecular hybridization tests of the surgical specimens fully met the diagnostic criteria of ENKTL-NT.

In the past medical history the patient refused to have lymphoma. Positive results for CD3, CD43, CD56, CD2, granzyme B, TIA-1, and EBER in tumor cells was consistent with primary intestinal NK/T-cell lymphoma. In addition, negative result for CD20 excluded a B-lymphocyte source. The positive Ki-67 labelling rate of $70 \%$ indicated a high degree of tumor malignancy, suggesting prognosis. Although CT and MRI examinations do not provide an accurate diagnosis, they can be used to evaluate patients for the development of regional lymph node and distant organ metastases. A study showed that nearly two-thirds of ENKTL-NT patients presented with localized stages I and II (22). As shown in Table 1, metastasis occurred in 5 of 9 patients, and lymph nodes were the main target. More notably, some cases of primary colon ENKTLNT are complicated by other serious colon diseases such as Crohn's disease and other colon tumors (e.g., colon adenocarcinoma), and their interrelationships are very confusing and need to be explored in further studies.
Treatment options for ENKTL-NT depend on the stage of diagnosis. Anthracycline-containing regimens, such as CHOP (cyclophosphamide, adriamycin, vincristine, prednisolone) or CHOP-like, are no longer conventional treatment because tumor cells acquire multidrug resistance (10). At present, satisfactory progress has been demonstrated in the treatment of early ENKTL-NT using radiotherapy combined with chemotherapy as the standard of care and it has largely improved the prognosis of patients as well as reduced tumor recurrence (23). However, there is no definite optimal treatment strategy for advanced ENKTL-NT. L-asparaginase is considered a key drug in the treatment of patients with advanced ENKTL-NT and is included in a variety of treatment regimens such as SMILE $(5,23)$. The P-GEMOX regimen is also considered an effective strategy for the treatment of advanced ENKTL-NT. A study reported that 35 newly diagnosed patients with stage III-IV, relapsed or refractory ENKTL-NT treated with 2-8 cycles of the P-GEMOX regimen had an overall remission rate of $80.0 \%$, with $51.4 \%$ of patients in complete remission. However, side effects included reduced white blood cells and impaired liver function (24). After systematic evaluation of the present patient by an oncologist the P-GEMOX treatment protocol was administered and the patient is doing well, with no signs of tumor recurrence, despite experiencing the described side effects. Hematopoietic stem cell transplantation and immunotherapy regimens for advanced ENKTL-NT are in prospective clinical studies, so a precise conclusion on their efficacy cannot be made at this time $(22,23,25)$. Overall, the outcome of primary ENKTL-NT of the colon is not very favorable, especially for patients diagnosed at an advanced stage. Most patients have a poor prognosis, with an average survival of only 2.83-9.5 months (19). Prediction of prognosis and treatment outcome is necessary to enable timely adjustment of the treatment plan based on response. A meta-analysis showed that plasma EBV-DNA positivity predicts patient prognosis (26). Other biomarkers can indicate a delayed response to immunotherapy in some patients treated with immunosuppressive drugs (27).

\section{Conclusions}

EBV infection is present in more than $95 \%$ of adults, which makes prevention of the cause of ENKTL-NT unlikely to be successful. The treatment and prognosis of patients is highly dependent on early diagnosis, which poses a challenge to clinicians, but is very necessary. The 
clinical manifestations of primary colonic ENKTL-NT are atypical; histopathological examination of ulcers found by colonoscopy requires more and deeper specimens. Where possible, plasma EBV-DNA tests should be performed. Unexplained bowel perforation requires thorough consideration by the clinician, and appropriate and reasonable exploration of the perforated bowel site for ulcers is necessary during surgery. Clinicians require more comprehensive understanding of ENKTL-NT to reduce misdiagnosis, and the discovery of relevant early markers and more effective treatment options are necessary.

\section{Acknowledgments}

Funding: This study was supported by the Science and Technology Research Project of The Education Department of Jiangxi Province, China (No. GJJ201523), and the Key Project of The First Affiliated Hospital of Gannan Medical University, China (No. YJZD202001).

\section{Footnote}

Reporting Checklist: The authors have completed the CARE reporting checklist. Available at https://dx.doi. org/10.21037/apm-21-3178

Conflicts of Interest: All authors have completed the ICMJE uniform disclosure form (available at https://dx.doi. org/10.21037/apm-21-3178). The authors have no conflicts of interest to declare.

Ethical Statement: The authors are accountable for all aspects of the work in ensuring that questions related to the accuracy or integrity of any part of the work are appropriately investigated and resolved. The study was approved by the Ethics Committee of the First Affiliated Hospital of Gannan Medical University (No. LLSC2021111201), and all procedures involving human participants followed the Declaration of Helsinki (as revised 2013). Written informed consent was obtained from the patient for publication of this case report and accompanying images. A copy of the written consent is available for review by the editorial office of this journal.

Open Access Statement: This is an Open Access article distributed in accordance with the Creative Commons Attribution-NonCommercial-NoDerivs 4.0 International License (CC BY-NC-ND 4.0), which permits the non- commercial replication and distribution of the article with the strict proviso that no changes or edits are made and the original work is properly cited (including links to both the formal publication through the relevant DOI and the license). See: https://creativecommons.org/licenses/by-nc-nd/4.0/.

\section{References}

1. Sui X, Zhang C, Jiang Y, et al. Wang S, Wang X. Resveratrol activates DNA damage response through inhibition of polo-like kinase 1 (PLK1) in natural killer/T cell lymphoma. Ann Transl Med 2020;8:688.

2. Kim WY, Montes-Mojarro IA, Fend F, et al. Epstein-Barr Virus-Associated T and NK-Cell Lymphoproliferative Diseases. Front Pediatr 2019;7:71.

3. Swerdlow SH, Campo E, Pileri SA, et al. The 2016 revision of the World Health Organization classification of lymphoid neoplasms. Blood 2016;127:2375-90.

4. Nagata H, Konno A, Kimura N, et al. Characterization of novel natural killer (NK)-cell and gammadelta T-cell lines established from primary lesions of nasal T/NK-cell lymphomas associated with the Epstein-Barr virus. Blood 2001;97:708-13.

5. Jeong SH. Extranodal NK/T cell lymphoma. Blood Res 2020;55:S63-71.

6. Haverkos BM, Pan Z, Gru AA, et al. Extranodal NK/T Cell Lymphoma, Nasal Type (ENKTL-NT): An Update on Epidemiology, Clinical Presentation, and Natural History in North American and European Cases. Curr Hematol Malig Rep 2016;11:514-27.

7. de Mel S, Hue SS, Jeyasekharan AD, et al. Molecular pathogenic pathways in extranodal NK/T cell lymphoma. J Hematol Oncol 2019;12:33.

8. Su YJ, Wang PN, Chang H, et al. Extranodal NK/ T-cell lymphoma, nasal type: Clinical features, outcome, and prognostic factors in 101 cases. Eur J Haematol 2018;101:379-88.

9. Chihara D, Oki Y. NK-Cell Lymphomas. Cancer Treat Res 2019;176:163-84.

10. Yamaguchi M, Oguchi M, Suzuki R. Extranodal NK/ T-cell lymphoma: Updates in biology and management strategies. Best Pract Res Clin Haematol 2018;31:315-21.

11. Kim HS, Lee DK, Baik SK, et al. Primary CD56+ T/NK cell lymphoma of the colon. J Gastroenterol 2002;37:939-46.

12. Zheng S, Xu H, Ouyang Q, Xue L, Zhang Y, Cui D. A case of rapid growing colonic NK/T cell lymphoma complicated by Crohn's disease. Chin J Cancer Res 
2013;25:119-23.

13. Tseng CE, Shu TW, Lin CW, et al. Synchronous adenocarcinoma and extranodal natural killer/T-cell lymphoma of the colon: a case report and literature review. World J Gastroenterol 2013;19:1850-4.

14. Mahuad CV, Bilbao ER, Garate GM, et al. Primary NK/ T cell lymphoma nasal type of the colon. Rare Tumors 2013;5:e9.

15. Aniwan S, Rerknimitr R, Wisedopas N, et al. Colonic NK/ T-cell lymphoma mimicking Crohn's disease. Endoscopy 2014;46 Suppl 1 UCTN:E312-E313.

16. Fares S, Lamchahab M, Aniba M, et al. Primary colonic extranasal NK/T-cell lymphoma: about a case. Pan Afr Med J 2017;26:112.

17. Li H, Lyu W. Intestinal NK/T cell lymphoma: A case report. World J Gastroenterol 2020;26:3989-97.

18. Duan Y, Huang J, Haybaeck J, et al. Primary extranodal natural Killer/T-cell lymphoma in a child in the colon: A case report. Medicine (Baltimore) 2021;100:e24232.

19. Zhang Y, Liu W, Zhang X, et al. Case Report: Primary NK/T Cell Lymphoma Nasal Type of the Colon With Multiple Intestinal Perforations. Front Oncol 2021;11:577939.

20. Yang L, Tang X, Peng X, et al. Clinical characteristics of primary intestinal NK/T cell lymphoma, nasal type: Case series and review of the literature. Pathol Res Pract 2018;214:1081-6.

Cite this article as: Xie Y, Liu Y, Wen C, Li W, Lu X, Deng L. Primary colon natural killer (NK)/T-cell lymphoma, nasal type, with perforations: a case report and literature review. Ann Palliat Med 2021;10(11):12025-12033. doi: 10.21037/apm21-3178
21. Chim CS, Au WY, Shek TW, et al. Primary CD56 positive lymphomas of the gastrointestinal tract. Cancer 2001;91:525-33.

22. Hu B, Oki Y. Novel Immunotherapy Options for Extranodal NK/T-Cell Lymphoma. Front Oncol 2018;8:139.

23. Suzuki R. NK/T Cell Lymphoma: Updates in Therapy. Curr Hematol Malig Rep 2018;13:7-12.

24. Wang JH, Wang L, Liu CC, et al. Efficacy of combined gemcitabine, oxaliplatin and pegaspargase $(\mathrm{P}$-gemox regimen) in patients with newly diagnosed advanced-stage or relapsed/refractory extranodal NK/T-cell lymphoma. Oncotarget 2016;7:29092-101.

25. Chan TSY, Li J, Loong F, et al. PD1 blockade with low-dose nivolumab in NK/T cell lymphoma failing L-asparaginase: efficacy and safety. Ann Hematol 2018;97:193-6.

26. Fei Q, Tian XK, Wu J, et al. Prognostic significance of Epstein-Barr virus DNA in NK/T-cell lymphoma: a metaanalysis. Onco Targets Ther 2018;11:997-1004.

27. Cho J, Kim SJ, Park WY, et al. Immune subtyping of extranodal NK/T-cell lymphoma: a new biomarker and an immune shift during disease progression. Mod Pathol 2020;33:603-15.

(English Language Editor: K. Brown) 